\title{
Relevanz, Wandel und Anforderungen wissenschaftlicher Politikberatung in der Raumplanung
}

Zimmermann, Karsten (2017): Relevanz, Wandel und Anforderungen wissenschaftlicher Politikberatung in der Raumplanung, disP - The Planning Review, 53:4, 21-32,

Abstract

Evidenced based planning is a salient issue and evokes questions about the role of expertise and scientific policy advice in spatial planning. The paper considers recent trends in the debate on scientific policy advice (crises of expertise, privatization/democratization of expertise, blurring of boundaries between science and policy) and reflects upon these trends against the background of the particular situation of scientific policy advice in spatial planning in Germany. Many insights from the wider debate on policy advice also hold true for spatial planning. However, spatial planning, at least in Germany, reveals some particularities. The scientific foundation of spatial planning is interdisciplinary and the gap between science and practice is probably less visible compared to other scientific disciplines. This makes dualistic approaches (science - policy boundaries) questionable. At the same time recent trends towards Gesellschaftsberatung (advice for society), transformative science and transdisciplinarity are attractive directions in spatial planning.

\section{1: Einleitung}

Die Frage nach der Integration wissenschaftlichen Wissens in Entscheidungsprozesse in der Raumplanung ist nicht zuletzt mit der Diskussion um evidence-based planning wieder virulent geworden (Faludi/Waterhout 2006; Davoudi 2006). Die Raumplanung gilt allerdings seit jeher als wissensintensive öffentliche Aufgabe, müssen doch für einen Planungsprozess verschiedene Formen technischen und sozialwissenschaftlichen Wissens integriert werden (Fürst 2010: 101; Rydin 2007; Nuissl 2000; Zimmermann 2010; Mazza 2002). Bisweilen wird Raumplanung als wissensbasierte Entscheidungsvorbereitung und damit als Politikberatung begriffen, die sich an Fachplanungen und gebietskörperschaftliche politische Entscheidungsträger richtet (siehe Beitrag Lendi). Wissenschaftliche Expertise ist zudem von besonderer Bedeutung für die Raumplanung, da sie aufgrund begrenzter Zuständigkeiten und mangelnder Durchsetzungskompetenzen gegenüber den Fachpolitiken und Adressaten auf persuasive Steuerung, gute Argumente und damit Wissen angewiesen ist (Fürst 2003; Sinz 2011). Wie Sinz (2011) bemerkt, müssen „für die Raumordnungspolitik eigene Paradigmen, Leitbilder, Ziele und Bewertungskriterien entwickelt werden, die auf gründlichen Analysen der räumlichen Verhältnisse und der Handlungsoptionen beruhen. Dies ist die Kernaufgabe der wissenschaftlichen Politikberatung auf dem Gebiet der Raumordnung und Raumentwicklung“ (ebd. 471). Berücksichtigt man die verschiedenen Funktionen, die Fürst (2010: 17-18) für die Raumplanung ausgemacht hat, so wird die Bedeutung von wissenschaftlicher Expertise insbesondere bei der Frühwarnfunktion (Einschätzung von (Umwelt-)Risiken), der Moderationsfunktion (Konfliktbewältigung) und der Orientierungsfunktion 
(Aufmerksamkeitssteuerung im Hinblick auf integrierte und gesamthaft räumlich orientierte Lösungen) deutlich. Kurzum: Die Praxis der Raumplanung ist beratungsintensiv (Fürst: 2010: 101).

Die Motivation für eine Inanspruchnahme wissenschaftlicher Beratung in der Raumplanung kann aber auch strategisch-instrumenteller Natur sein. Schließlich kann durch die Einsetzung von Expertengremien oder die Beauftragung von Gutachten eine implizite Delegation von schwierigen Entscheidungen erreicht werden. Zumindest eine bewusste Aufmerksamkeitsverschiebung, eine Versachlichung und Neutralisierung eigentlich politisch zu entscheidender Sachverhalte, eine gewünschte Verzögerung oder auch Akzeptanzgewinnung durch wissenschaftliche Expertise sind durchaus gewünschte Effekte wissenschaftlicher Politikberatung (ebd.: 102). Damit ist die vielfach beschriebene gleichzeitige Politisierung der Wissenschaft und Verwissenschaftlichung der Politik auch in der Raumplanung offenbar relevant. Es ist die Absicht dieses Beitrags, die für die wissenschaftliche Politikberatung allgemein gemachten Beobachtungen im Kontext der Raumplanung zu reflektieren. Der Beitrag beginnt mit einer Definition und Darstellung der Entwicklung der wissenschaftlichen Politikberatung und geht dabei auf Entwicklungen in der Raumplanung ein. Anschließend werden Konsequenzen der jüngeren Trends diskutiert und abschließend auf die Rolle staatlicher Wissensproduktion eingegangen. Es zeigt sich, dass klassische wissenschaftliche Politikberatung in der Raumplanung unverzichtbar ist, eine Diskussion über die Qualitätskriterien und den Sondercharakter des Beratungswissens sowie zur Grenzarbeit zwischen Wissenschaft und Politik aber wünschenswert wäre. Die Raumplanung kann auch von Veränderungen in Richtung einer allgemeiner als Gesellschaftsberatung gefassten öffentlichen Wissensproduktion profitieren und ihren gesellschaftspolitischen Stellenwert stärken (science going public).

Auch wenn es nicht an jeder Stelle explizit gemacht wird, so bildet doch die deutsche Situation den Bezugspunkt der hier gemachten Überlegungen. Die Aussagen sind also im internationalen Vergleich zu relativieren.

\section{Was ist wissenschaftliche Politikberatung?}

Die für die Raumplanung relevante Wissensproduktion findet mittlerweile an vielen Orten statt und wird durch viele verschiedene private und öffentliche Akteure geleistet. Nicht immer handelt es sich dabei um wissenschaftliche Politikberatung, sondern um andere Formen der kooperativen Wissensgenerierung und des Wissenstransfers. Neben der Privatisierung der Politikberatung ist hier vor allem auf die Diskussion zur transdisziplinären oder transformativen Wissenschaft oder auch der partizipativen Politikberatung hinzuweisen. Auf diese relativ neuen Formen der Produktion politisch relevanten Wissens sowie auf weitere Formen der Gesellschaftsberatung wird später eingegangen. Zunächst soll an dieser Stelle ein eng gefasster Begriff der wissenschaftlichen Politikberatung eingeführt werden, der sich von den genannten Formen des Wissenstransfers unterscheidet.

Schützeichel beschreibt Beratungsprozesse allgemein als „thematisch bzw. sachlich auf Entscheidungsprobleme fokussierte, zeitlich limitierte Kommunikation zwischen einem 
Alternativen offerierenden Ratgeber und einem um Entscheidungen ringenden Ratsuchenden (2008: 78). Es handelt sich demnach auch bei der wissenschaftlichen Politikberatung um eine besondere Form der Kommunikation, die durch eine asymmetrische Beziehung gekennzeichnet ist. Die Beratungsbeziehung besteht aus einem über Wissen verfügenden Auftragnehmer und einem Auftraggeber, der ein Wissensdefizit hat und dieses Defizit ausgleichen will. Umfang und Ziel der Beratung sind in der Regel klar definiert. Es kann sich um Prognosen, Szenarien (also Produkte mit eher deskriptivem Charakter, wenn auch mit Frühwarnintentionen und somit Entscheidungszumutungen verbunden), Handlungsempfehlungen mit klar instrumentellem Bezug, Bewertung von Alternativen bei der Erreichung eines politischen Ziels, Folgenabschätzungen oder Evaluationen handeln (Lentsch 2008: 195). In der Raumplanung wie auch in anderen Politikfeldern geht wissenschaftliche Politikberatung über die sachliche Fragestellung (Planinhalte, Raumentwicklung) oft hinaus und schließt die Bewertung von Instrumenten und Organisationsformen mit ein (z.B. Evaluation der Städtebauförderung, vgl. Altrock 2016). Wissenschaftliche Politikberatung setzt eine vergleichsweise neutrale Position des beratenden Wissenschaftlers voraus. D.h. idealerweise verfolgt der Experte keine Eigeninteressen und ist methodisch der Wahrheitsfindung verpflichtet. Als Berater fungieren Wissenschaftler oder Experten als Träger des Expertenwissens, das nach wissenschaftlichen Methoden gewonnen und bewertet wurde. Experten sind durch wissenschaftliche Ausbildung zertifiziert und in der Regel an einer wissenschaftlichen Einrichtung beschäftigt. Häufig haben sich feste Verbindungen zwischen Berater und Beratungsempfänger etabliert und sind in Form von Sachverständigenräten, Beiräten, Kommissionen oder öffentlichen Instituten institutionalisiert. Das Beratungsverhältnis sieht eine klare Rollenverteilung vor, wobei Rollenkonflikte nicht auszuschließen sind, wenn Wissenschaftler als Mit-Entscheider auftreten (wollen) oder mit dieser Absicht eingebunden werden. Beratungswissen zielt auf die Analyse und die Beschreibung von Sachverhalten sowie auf die Entwicklung von Handlungsempfehlungen. Ob aus der Beschreibung von Sachverhalten ein gesellschaftliches Problem wird, dass politisch zu adressieren wäre, muss aber nicht Teil der Beratung sein, sondern kann politischer Kommunikation überlassen bleiben (Saretzki 2005). Wissenschaftliche Politikberatung ist insofern abzugrenzen von Lobbyismus oder sonstigen Prozessen der öffentlichen Meinungsbildung.

Allgemeine Politikberatung unterscheidet sich von wissenschaftlicher Politikberatung durch die beteiligten Akteure. Nicht jede Form der Produktion politisch relevanten Wissens kann als wissenschaftliche Politikberatung bezeichnet werden. Vielmehr ist gerade in den letzten Jahren das Konzept wissenschaftlicher Politikberatung gezielt herausgefordert worden. Zum einen beinhalten Konzepte der Gesellschaftsberatung (Leggewie 2006), der transdisziplinären oder transformativen Wissenschaft oder der partizipativen Politikberatung ein verändertes Verhältnis von Wissenschaft, Politik und Zivilgesellschaft (Nowotny et al. 2001; Schneidewind/Singer-Brodowsky 2013). Unter dem in diesem Sinne sehr weiten Begriff der Politik- oder Gesellschaftsberatung wird die Vermittlung wissenschaftlicher Erkenntnisse durch Think Tanks, Stiftungen, NGO,s und Medien verstanden (Schützeichel 2008; Leggewie 2006). Zielgruppe ist im weitesten Sinne die Gesellschaft vor dem Hintergrund bestimmter Problemlagen (Klimawandel, Migration, Energiewende, etc.). Zum anderen hat sich ein 
Beratungsmarkt herausgebildet, der von privaten Anbietern, aber auch Stiftungen geprägt wird. Ziel ist nicht selten politisches Agenda-Setting. Insbesondere außeruniversitäre Institute, aber auch Stiftungen interpretieren ihren Beratungsauftrag bewusst weit und haben viele, mitunter diffuse Zielgruppen im Blick.

Wissenschaftliche Politikberatung im engeren Sinne richtet sich aber an das Parlament oder die Exekutive. Sie ist - trotz aller Kritik und Skepsis - nötig, weil in Verwaltungen und Parlamenten nicht immer der nötige Sachverstand für die Lösung komplexer Probleme vorausgesetzt werden kann. Zudem besteht nach wie vor der Glaube, durch eine breitere Wissensbasis, methodisch abgesicherte Erkenntnisse und sonstige Expertise die Rationalität von Entscheidungen erhöhen zu können und ggf. auch Konflikte besser handhaben zu können. Wie Anna Geiss am Beispiel des Mediationsverfahrens für den Ausbau des Frankfurter Flughafens zeigen kann, ist die tatsächliche Reichweite dieser Annahme fallweise zu prüfen (Geiss 2008). Die Trennung von Sachlogik und politischer Rationalität ist gerade bei projektbezogenen Verfahren wie dem Raumordnungsverfahren, wo Gewinner und Verlierer klar identifiziert werden können, nur schwer durchzuhalten. In der Tat ist die Wissensgrundlage derartiger Verfahren häufig ein Streitpunkt. Als Reaktion auf ein Gutachten etwa zum Bedarf einer neuen Bahntrasse wird dann gezielt ein Gegengutachten in Auftrag gegeben, was mitunter die Glaubwürdigkeit von Expertise insgesamt schmälert.

Die Annahme, durch eine Verwissenschaftlichung der Politik zu besseren Entscheidungen und effektiveren Lösungen zu kommen, ist eng mit der Geschichte der Raumplanung verknüpft. Insbesondere Schelsky (1965) wird nach wie vor zitiert, um die für die Zeit der 1960er und frühen 1970er Jahre dominierende Arbeitsteilung zwischen Wissenschaft und politischer Praxis zu beschreiben (Schützeichel 2008: 17). Demnach sollten Parlamente und Verwaltungen weitgehend dem objektiven Sachverstand der Experten folgen, deren Kapazitäten gezielt ausgebaut wurden. In der Zeit der Planungseuphorie der 1960er und 1970er Jahre sollte eine politische Logik des Entscheidens der wissenschaftlich-technischen Logik untergeordnet werden, die vielleicht mit dem Anspruch an Genauigkeit und an technokratisch-methodischer Beherrschbarkeit von Wertfragen überfordert war. Der Spielraum der Parlamente und Regierungen sank zunächst erheblich, was zur späteren Ablehnung der Herrschaft der Experten führte und zugleich einer der Gründe für das Scheitern der integrierten Entwicklungsplanung war (Blotevogel 2005). Trotzdem ist wissenschaftliche Politikberatung nach wie vor im Kontext einer doppelten Legitimationsanforderung zu betrachten (Weingart/Lentsch 2008: 11). Neben der sachlichen Angemessenheit einer Entscheidung ist auch die Zustimmungsfähigkeit letztlich auf wissenschaftliche Expertise angewiesen. Ignoranz der Politik gegenüber den Erkenntnissen der Wissenschaft birgt das Risiko schwindender Legitimation.

\section{Wandel der Politikberatung}

Im vorangehenden Abschnitt wurden bereits einige Veränderungen der wissenschaftlichen Politikberatung kurz angesprochen. Sie sollen hier noch einmal gebündelt dargestellt werden. Ausgangspunkt ist das duale Modell, das bereits von Renate Mayntz (1994) kritisch diskutiert wurde und gegenwärtig um so mehr infragegestellt wird. Das duale Modell unterscheidet mit 
der Wissenschaft und der politisch-administrativen Praxis zwei Sphären, die relativ autonom operieren. Die Wissenschaft folgt der Wahrheitssuche, die Politik wesentlich dem Machterhalt. Wissenschaftler sind es gewohnt neue Wissensoptionen zu öffnen, Politiker sind dagegen eher an einer Schließung von Optionen interessiert und erwarten eindeutige Ergebnisse und keine Ambivalenzen, da sie auf Entscheidungen zuarbeiten (Weingart/Lentsch 2008: 13). Ein linear gedachter Transfer wissenschaftlichen Wissens in die Sphäre der Praxis scheint angesichts vielfältiger Verflechtungen nicht mehr zeitgemäß. Vielmehr wird Politikberatung als rekursiver Prozess beschrieben (Böcher 2007: 25f.). Im Folgenden werden überblicksartig die wichtigsten Entwicklungen aus der Diskussion zur wissenschaftlichen Politikberatung zusammengefasst und auf die Situation der Raumplanung bezogen.

\section{Trend 1: Grenzen und Grenzverwischungen}

Prägend für die deutsche Diskussion zur wissenschaftlichen Politikberatung sind nach wie vor die Ergebnisse des von Ulrich Beck in den 1980er Jahren initiierten DFGSchwerpunktprogramms zur Verwendung sozialwissenschaftlichen Wissens (Beck/Bonß 1989). Eine der wesentlichen Einsichten der aus diesem Verbund resultierenden Verwendungsforschung war, dass ein direkter Zusammenhang von wissenschaftlicher Politikberatung und erfolgreicher (oder erfolgreich veränderter) politischer Praxis kaum zu beobachten war. Vielmehr war ein Verschwinden wissenschaftlichen Wissens im Kontext praktischer Politik festzustellen (ebd.). Implizit ging der damalige Forschungszusammenhang allerdings davon aus, dass es eine Sphäre der Produktion wissenschaftlichen Wissens und eine (politische) Sphäre der Anwendung gäbe. Beide Sphären wurden als mehr oder weniger getrennt und unterschiedlichen Logiken folgend gedacht. Derzeit verweisen aber nicht wenige Autoren auf die gleichzeitige Verwissenschaftlichung der Politik und Politisierung der Wissenschaft (Mayntz 1994; Maasen/Weingart 2005; Lendi 2005; Weingart/Lentsch 2008; Straßheim 2013). Durch die Verwischung der Grenzen und immer enger werdende Verflechtungen der beiden Sphären wird es schwieriger, den Einfluss der Politik auf die Wissenschaft und umgekehrt den Einfluss der Wissenschaft auf die Politik eindeutig zu identifizieren.

Stand der allgemeinen Diskussion bleibt, dass wissenschaftliches Wissen seinen Charakter als wissenschaftliches Wissen beim Übertritt in die Sphäre der politischen Praxis verliert und in eine Metamorphose eintritt (Saretzki 2005; Beck/Bonß 1989). Anstelle der wissenschaftlichen Logik der Entdeckung tritt eine Logik der Verwendung. Oft verschwindet wissenschaftliches Wissen im Alltag der Politik komplett oder wird stark vereinfacht. Rob Hoppe (2005) weist nun darauf hin, dass die Verwischung der Grenze zwischen Wissenschaft und Politik dazu führt, dass seitens der Politik und der Wissenschaft immer wieder aktive Bemühungen unternommen werden, um genau diese Grenze wieder wirksam werden zu lassen. Die Wissenschaft benötigt die Grenzziehung zwischen Wissenschaft und anderen Gesellschaftsbereichen, um weiterhin den Anspruch einer privilegierten Sphäre der Produktion von als gültig anerkannten Wissen erheben zu können. Auf diese Weise anerkanntes Wissen kann wiederum für die Rechtfertigung politischer Entscheidungen genutzt werden (Weingart/Lentsch 2008). Aber gerade diese Verwendung wissenschaftlichen Wissens zwingt die Wissenschaft geradezu zu identitätsbehauptenden Grenzziehungen. Im Resultat führt die kontinuierliche Grenzarbeit 
zwischen Wissenschaft und Politik dazu, dass Rollen stets neu ausgehandelt werden, um die Definitionsmacht im Beratungsprozess über Strategien des Autonomieerhalts zu erhalten bzw. zu stärken (Korinek/Veit 2013). Als Konsequenz wurde die Grenzarbeit an der Schnittstelle zwischen Wissenschaft und Politik thematisiert und es wurden - in Anlehnung an Gieryn (1998) - „,boundary arrangements“ bzw. „boundary organizations“ als neue Kategorie eingeführt (Hoppe 2005; Halffman/Hoppe 2005; Straßheim 2013, 2008; Guston 2001), in deren Mittelpunkt häufig wissenschaftliche Einrichtungen mit öffentlicher Finanzierung stehen, die nahe an der Exekutive sind (siehe Beitrag von Feiertag).

In der wissenschaftlichen Politikberatung im Bereich der Raumplanung ist es in den letzten Jahren wiederholt zu derartigen Grenzverschiebungen und temporären Klärungen gekommen (siehe Beitrag Feiertag; Pahl-Weber 2011). Konkrete Resultate von Grenzveränderungen können Zusammenlegungen von Instituten, Neugründungen von Agenturen wissenschaftlicher Politikberatung als autonome Einheiten oder aber der Aufbau interner Kapazitäten in einem Ministerium sein. Fraglich ist, in welche Richtungen die Grenzverschiebungen jeweils genau verlaufen und welche Folgen dies für die Relevanz der wissenschaftlichen Politikberatung hat.

\section{Trend 2: Krise der Expertise}

Ein weiterer Trend ist in der weit verbreiteten Skepsis gegenüber wissenschaftlicher Expertise und wissenschaftlicher Politikberatung zu sehen (Maasen/Weingart 2005; Saretzki 2005, 1997). Die Gründe dafür sind vielfältig und reichen von der stets vermuteten Instrumentalisierung und Manipulation der Wissenschaft durch die Politik bis hin zu epistemischen und normativen Grenzen in der Wissenschaft selbst (Weingart/Lentsch 2008: 12-14). Die Qualitäten der Eindeutigkeit und Gesichertheit des wissenschaftlichen Wissens, die für die Nutzung als Legitimationsressource politischer Entscheidungen gefordert werden, scheinen nicht mehr gegeben zu sein. Saretzki fasst mit Blick auf die Begrenztheit der Expertise vier Aspekte zusammen (Saretzki 2005: 348-352):

1) Die Spezialisierung bzw. fachwissenschaftliche Kompetenz der wissenschaftlichen Disziplinen in einem hochgradig differenzierten Wissenschaftssystem steht Problemen gegenüber, die integrierte Lösungen erfordern und daher die Grenzen der Disziplinen überschreiten (fachwissenschaftliche Grenze). Problematisch ist aus Sicht von Saretzki, dass fachwissenschaftliche Experten angesichts interdisziplinärer Problemlagen eigentlich ihren Kompetenzbereich überschreiten (ebd. 349). Dies ist für die Raumplanung äußerst relevant, da sich die Raumplanung als Querschnittsaufgabe versteht und für integrierte Lösungen eintritt (s.u.). Zudem hat sich die Raumplanung in den vergangenen Jahren nicht zufällig an der Nachhaltigkeitsforschung und der dort praktizierten Interdisziplinarität orientiert (Blotevogel/ Wiegand 2015).

2) Die Erkenntnisgrenze: wissenschaftliches Wissen kann nicht mehr den Status gesicherter Erkenntnis beanspruchen, es hat vielmehr hypothetischen Charakter. Aussagen über Risiken in Form von Prognosen, Szenarien oder Modellierungen sind mit klaren Regeln verknüpft, die wiederum Einschränkungen und Bedingungen enthalten, unter denen die Aussagen gelten. 
Gleichwohl werden Entscheidungen bei Akzeptanz der begrenzten Reichweite wissenschaftlicher Aussagen getroffen (hard decisions with soft evidence). Zu erwähnen ist auch die zunehmend anerkannte Komplexität und Interdependenz, die sowohl an der Zahl der zu berücksichtigenden Faktoren als auch an der Dichte der zwischen den Faktoren bestehenden Kausalbeziehungen gemessen werden kann. Dies verändert die Handlungsgrundlagen vorausschauender Planung. Sicher kann infragegestellt werden, ob dieses Argument seit der Diskussion zur rationalen Planung der 1960er und 1970er Jahre noch etwas qualitativ Neues beschreibt. Angesichts der bleibend hohen Zahl an Beiträgen, die sich mit der Bewältigung von Problemen zweiter Ordnung, nicht intendierten Nebenfolgen und dem Problem der Komplexität und des Nicht-Wissens beschäftigen, scheint es angemessen, dieses Argument beizubehalten (de Roo et al. 2012).

3) Die normative Grenze verweist auf die Abhängigkeit relevanter wissenschaftlich informierter Problemdefinitionen von gesellschaftlichen Problemwahrnehmungen und Werten. D.h. die Identifizierung einer gesellschaftlich relevanten Problemstellung ist stets eine Vermischung aus der wissenschaftlichen Beschreibung eines zunächst unproblematischen Sachverhalts und der dann darauf bezogenen gesellschaftlichen Kommunikation, die aus dem Sachverhalt ein zu bearbeitendes Problem macht. Wissenschaftler erweisen sich dann häufig als Grenzgänger. Viele wissenschaftliche Erkenntnisse geraten aber erst gar nicht in diesen Prozess.

4) Die vierte Grenze verweist auf das Auseinanderfallen von Erkenntnisansprüchen mit universaler Geltung und lokaler Kontextualisierung. Abstrakt formuliertes Wissen muss in lokale Wirkungszusammenhänge eingebettet werden, soll es sich als wirksam und richtig erweisen. Es wird dann in der Regel mit einem lokalen Kontextwissen verknüpft, was durchaus zu Konflikten zwischen wissenschaftlich als gesichert geltenden Aussagen und lokalen Erfahrungen führen kann (Fischer 2000). Auch dies ist für die Raumplanung von hoher Relevanz.

\section{Trend 3 Vervielfältigung der Akteure und Quellen von Wissen}

Gerade die Krise der Expertise hat nun paradoxerweise statt zu einer Abnahme wissenschaftlicher Politikberatung $\mathrm{zu}$ einer anhaltenden Zunahme von Gutachten, Begleitforschungen und Verfahren der Politikberatung geführt (Lendi 2005; Weingart/ Lentsch 2008). Das Resultat ist eine erhebliche Pluralisierung der Wissen produzierenden privaten und öffentlichen Akteure (Weingart/Lentsch 2008: 12). Die Wissenschaft (im Sinne von Akademien, Max-Planck-Instituten, Universitäten) ist nicht mehr allein der Ort der Generierung politisch relevanten Wissens. Neben die Wissenschaft sind Think Tanks, NGOs, Stiftungen, freie Institute wie die Öko-Institute und privatwirtschaftliche Beratungsunternehmen oder aber citizen science-Bewegungen getreten. Die Öffnung des Beratungssystems in Richtung Interdisziplinarität und Bürgergesellschaft (science going public, Mode 2-Wissensproduktion, transformative Wissenschaft) hat ebenfalls zur Vervielfältigung der Akteure beigetragen (Nowotny et al. 2001; Schneidewind/Singer-Brodowsky 2013). 
Sicher hat es auch in der Raumplanung eine Vervielfältigung der Akteure gegeben, die auf einem Wissensmarkt für die Raumplanung relevantes Wissen oder darauf bezogene Dienstleistungen anbieten. Neben die bekannten Akademien, Universitäten, Institute und Ressortforschungseinrichtungen (und Programme) sind private Organisationen getreten, die sich um öffentlich finanzierte Auftragsforschung bemühen oder Expertise im Auftrag privater Akteure erstellen. Nicht unerwähnt bleiben sollten die Stiftungen, die zum Teil eher vermittelnde Funktionen übernehmen, zum Teil aber auch als advokatorische Think Tanks fungieren und eigene Positionen einbringen, die wissenschaftlich begründet werden (etwa (Bertelsmann-Stiftung, Wüstenrot-Stiftung, Schader-Stiftung, Denkwerkzukunft). Diese beteiligen sich an öffentlichen Diskussionen und zum Teil auch an Ausschreibungen, so dass hier von Konkurrenzsituationen gesprochen werden kann. In Deutschland spielen advokatorische Think Tanks, die mit eigenen Positionen die Diskussion beleben, insgesamt aber eine eher untergeordnete Rolle. Ein Beispiel für einen advokatorischen Think Tank in den USA ist das Lincoln Institute for Land Policy ${ }^{1}$ oder das Brookings Institute, in dem Bruce Katz das Metropolitan Policy Programme leitet. ${ }^{2}$ In der Schweiz wäre an Avenir Suisse zu denken. ${ }^{3}$

\section{Spezifische Entwicklungen der Politikberatung in der Raumplanung}

Zweifellos kann auch für den Bereich der Raumplanung von der Herausbildung einer eigenständigen Sphäre der Politikberatung gesprochen werden, in der sich private Agenturen, Stiftungen und öffentliche Forschungseinrichtungen in vielfältiger Weise am Wissensmarkt beteiligen. Viele der zuvor genannten Trends sind also auch im Feld der Raumplanung relevant. Zugleich ist die Raumplanung in einer Sondersituation, sodass einige der Trends konkretisiert und spezifische Entwicklungen beschrieben werden müssen. Politikberatung in der Raumplanung folgt kaum einem einheitlichen Prinzip. Je nach Ebene und Aufgabe zeigen sich deutliche Unterschiede im Hinblick auf die Akteure als auch die Inhalte: während auf Bundesund zum Teil auch auf Landesebene ${ }^{4}$ Beratungsformate fest etabliert oder gar gesetzlich gefordert sind (Beirat für Raumordnung, Akademie für Raumforschung und Landesplanung, Bundesinstitut für Bau-, Stadt- und Raumforschung, Deutsches Institut für Urbanistik und weitere raumwissenschaftliche Institute wie Institut für ökologische Raumentwicklung, Institut für Landes- und Stadtentwicklungsforschung, Institut für Regionalentwicklung und Strukturplanung und auch universitäre Institute), kommen auf regionaler und lokaler Ebene auch andere Formen zum Einsatz (Gutachten, Expertengespräche zu ausgewählten Themen, wissenschaftliche Begleitung von Projekten). Im Bereich der Raumplanung existiert keine Einrichtung wie das Büro für Technikfolgenabschätzung beim Deutschen Bundestag, das RobertKoch-Institut oder das Bundesinstitut für Risikobewertung. Diese Einrichtungen haben jeweils ein klares Mandat und von ihnen wird erwartet, führend in der wissenschaftlichen Politikberatung zu sein. Die Situation in der Raumplanung ist heterogener.

\footnotetext{
${ }^{1}$ www.lincolninst.edu/

${ }^{2}$ www.brookings.edu/about/programs/metro, vgl. Katz/Bradley 2013

${ }^{3}$ www.avenir-suisse.ch

${ }^{4}$ Vgl. den kurzen Überblick bei Fürst 2010: 103
} 
Zudem erfordern die verschiedenen Sachverhalte und Themen der Raumplanung unterschiedliche Formen der Politikberatung. Prognosen und Situationseinschätzungen im Hinblick auf die Bevölkerungsentwicklung oder die Folgen des Klimawandels sind dem Charakter nach andere Beratungsmaterien als Empfehlungen und Begleitforschungen, die sich auf Planungsprozesse (Kommunikation, Moderation) oder neue Instrumente, rechtliche Regelungen oder gar Grundsätze der Raumordnung beziehen. In der Raumplanung spielen daher insbesondere zwei der von Saretzki genannten Grenzen eine Rolle:

1) Aufgrund der ausgeprägten Interdisziplinarität der Planungswissenschaften ist eine besondere Situation entstanden, die sich auch bei der Generierung und Bewertung von Beratungswissen bemerkbar macht. Die Bewertung wissenschaftlicher Exzellenz in den Planungswissenschaften orientiert sich an den Maßstäben und Kulturen mehrerer Disziplinen, (Rechtswissenschaften, Geographie, Ökonomie, Naturwissenschaften, Städtebau, Sozialwissenschaften). Da wie geschildert in einem Beratungsprozess in der Raumplanung mal juristische Expertise, mal wirtschaftsgeographische Analysen oder aber eher sozialwissenschaftliche Begleitforschung gefragt sind, unterscheiden sich Beratungsprozesse und die Kriterien für die Bewertung der Qualität des Beratungswissens. Hinzu kommt, dass in der Raumplanung auch Beratungsleistungen anderer Handlungsfelder für die Praxis genutzt werden (müssen), da sie für Fragen der Raumentwicklung unmittelbar relevant sind. Dies gilt in erster Linie für den Bereich der Umweltpolitik und den Naturschutz. Die Gutachten und Veröffentlichungen des Sachverständigenrats für Umweltfragen werden von Planungswissenschaftlern und Praktikern aufmerksam verfolgt und häufig gezielt zur Stärkung der eigenen Position genutzt. Zudem werden die Ergebnisse von Enquete-Kommissionen des Bundestages zur Kenntnis genommen (etwa zum bürgerschaftlichen Engagement, zur nachhaltigen Energieversorgung und zum Klimawandel).

2) Des weiteren spielt die zeitliche und räumliche Varietät der Raumentwicklung eine Rolle. Dies wurde zuletzt im Zusammenhang mit der Klimaanpassung und dem demographischen Wandel immer wieder deutlich gemacht. Szenarien und Prognosen sind hier das Mittel der Wahl. Dabei wird mit unterschiedlichen Zeithorizonten gearbeitet, die von eher kurzfristigen bis zu langfristigen Szenarien reichen können (2020, 2050, 2070). Diese Methodik ist mit Unsicherheiten und räumlicher Vielfalt im Hinblick auf Eintrittswahrscheinlichkeiten konfrontiert (Gramelsberger 2009). Gerade bei der regionalen Betroffenheit durch den Klimawandel ergeben sich erheblich räumliche Unterschiede. Räumliche Varietät ist auch im Hinblick auf Planungssysteme zu berücksichtigen, was nicht zuletzt auf europäischer Ebene immer wieder deutlich wird. Je nach Planungssystem ergeben sich andere Optionen und Handlungsmöglichkeiten, was gerade in den föderal organisierten oder stark regionalisierten Staaten (wie Italien, Vedeli 2012) deutlich wird. Die von Saretzki angesprochenen lokalen Kontextbedingungen als Grenze der Anwendbarkeit von wissenschaftlicher Expertise mit universellem Geltungsanspruch sind also in der Raumplanung hervorzuheben.

3) Die Planungswissenschaften sind durch ihre Praxis- und Politiknähe gekennzeichnet. Die Etablierung der Disziplin und der damit verknüpften Studiengänge an den Universitäten ist eng verknüpft mit der Phase der Steuerungseuphorie der 1960er und 1970er Jahre und hatte somit 
einen klaren Bezug zur Praxis, die diese Expertise nachfragte. Dies macht sich insofern bemerkbar, als mehr oder weniger jede wissenschaftliche Analyse auch jenseits der unmittelbaren Beratungspraxis die Bewertung vergangener oder aktueller Planungspraktiken beinhaltet (anders als in den Naturwissenschaften). Damit ist häufig eine hohe Praxisrelevanz auch in der Grundlagenforschung gegeben. Raumplanungsforschung findet nicht im Labor oder unter experimentellen Bedingungen statt, sondern in der Regel in Städten und Regionen unter Beteiligung lokaler Akteure (Pahl-Weber 2011). Eine eindeutige Abgrenzung der Sphäre der Planungswissenschaften von der Sphäre der Praxis ist aus diesem Grunde schwierig und auch gar nicht erwünscht. Gegenwärtig müssen sich jedoch auch Planungswissenschaftler an Universitäten und Leibniz-Instituten an den wissenschaftlichen Exzellenzkriterien messen lassen, was den Bereich der Politikberatung in der Tendenz unattraktiv macht. Man überlässt dieses Feld eher anderen Akteuren.

4) Erwähnenswert ist nicht zuletzt die Europäisierung der Wissensgenierung in der Raumplanung und Raumentwicklungspolitik. Mit dem ESPON-Programm unterstützen die Mitgliedstaaten seit 2002 die Generierung und den Transfer wissenschaftlichen Wissens (territorial evidence). ESPON ist formal weitgehend unabhängig von der Europäischen Kommission. Gleichwohl wird der Anspruch erhoben, politikrelevantes Wissen zu erzeugen (evidenced based policy) und insbesondere dem Parlament und den Kommissionsdienststellen zur Verfügung zu stellen. Die Organisation des ESPON-Programms lässt allerdings eine bedarfsgenaue Steuerung der Wissensproduktion wie etwa bei der Ressortforschung kaum zu. ESPON hat einen eher globalen, aber nicht unbedingt handlungsorientierten Fokus. Die Wirkung lässt sich trotz erheblicher Bemühungen der ESPON-Geschäftsstelle nur schwer einschätzen. Zweifellos finden Ergebnisse der im Rahmen von ESPON finanzierten Forschungsprojekte Eingang in Policy-Dokumente wie etwa den Kohäsionsberichten. Eine präzisere Beschreibung der Wirkungen des ESPON-Programms kann aber hier nicht vorgenommen werden. Noch schwieriger ist eine Einschätzung der Wirkung auf Ebene der Mitgliedstaaten.

\section{Anforderungen an die Politikberatung in der Raumplanung}

Aus den bisher beschriebenen Entwicklungen ist deutlich geworden, dass sich Beratungsprozesse und Entscheidungen über Prioritäten in der Forschung in Verhandlungsnetzwerke verlagern, die eben nicht eindeutig dem Sektor Wissenschaft oder Politik zu zuordnen sind. An dieser Stelle kann nicht bewertet werden, ob dies zu einer eindeutigen Politisierung der Wissenschaft oder Verwissenschaftlichung der Politik führt. Eine solche Diagnose wäre je nach Handlungsfeld zu präzisieren und würde in der für Politisierung anfälligen Klima- und Umweltforschung anders ausfallen als in der Molekularbiologie. Zudem ist davon auszugehen, dass beide Prozesse bis zu einem gewissen Grad parallel ablaufen. In jedem Fall kann festgehalten werden, dass diese Entwicklung aus der Sicht der Raumplanung alles andere als unbekannt ist. Allerdings hat in der Raumplanung kein Diskurs zur organisatorischen und prozessualen Ausgestaltung der wissenschaftlichen Politikberatung stattgefunden. Auf der Grundlage der bisherigen Ausführungen lassen sich die folgenden Anforderungen formulieren.

\subsection{Beratungswissen als Sonderwissen ausflaggen und anerkennen}


Das Beratungswissen als Gegenstand eines Beratungsprozesses kann als Sonderwissen bezeichnet werden (Weingart/Lentsch 2008: 20), denn „Es besteht auch weitgehend Einigkeit darüber, dass die Angemessenheit der im Beratungsprozess kommunizierten Expertise nicht allein im Hinblick auf die wissenschaftliche Richtigkeit beurteilt werden muss, sondern auch im Hinblick auf die Verwertbarkeit für politische Entscheidungen" (ebd.: 40).

Politikberatung ist somit eine eigenständige Form der Wissensproduktion und sollte als solche Anerkennung finden. Sheila Jasanoff (1990) hat schon recht früh mit dem Begriff der „regulatory science" auf die Besonderheiten des Beratungswissens aufmerksam gemacht. Neben der epistemischen Robustheit (wissenschaftliche Qualität) sind auch die politische Robustheit und ggf. die soziale Robustheit als Qualitätskriterium zu berücksichtigen. Die politische Robustheit impliziert sowohl die Legitimität als auch die Sachangemessenheit der Expertise. Die wissenschaftliche Gültigkeit des Wissens, auf das sich politische Akteure beziehen, schafft Legitimität für Entscheidungen. Zugleich verspricht Beratungswissen rationale Problemlösungen (Effektivität). Mit dem Konzept der sozialen Robustheit wird auf die Notwendigkeit der sozialen Kontextualisierung abstrakt gewonnenen Wissens durch den Einbezug und die Akzeptanz gesellschaftlicher Interessen und Perspektiven im Beratungsprozess Bezug genommen, was eine Erweiterung des Akteurskreises durch Partizipation zur Voraussetzung hat. Beratungswissen muss also robust in dem Sinne sein, dass es sich in sozialen und politischen Anwendungskontexten als funktions- und anschlussfähig erweist. Damit werden andere Bedingungen an die Wissensgenerierung in der Politikberatung formuliert, als wir sie im Bereich der Grundlagenforschung kennen. Sie muss inklusiver sein und den Transfer gewährleisten. Dies hat Folgen für die Qualitätssicherung, was wiederum Verfahrens- und Organisationsfragen aufwirft. Da die uns bekannten Verfahren der wissenschaftlichen Qualitätssicherung wie das peer-review Verfahren für die Bewertung der Qualität des Beratungswissens nicht angemessen sind, wird eine eigenständige Form der Qualitätskontrolle und Evaluation benötigt, die sich von denen der rein wissenschaftlichen Erkenntnisproduktion unterscheidet (Weingart/Lentsch 2008; vgl. für das Beispiel der Evaluation der Akademie für Raumforschung und Landesplanung: Blotevogel/Wiegand 2015; siehe auch Saretzki 2005: 348).

\subsection{Qualitätssicherung in der Politikberatung}

Justus Lentsch schlägt vor, Qualitätskriterien in Bezug auf Beratungswissen produkt- und prozessbezogen zu formulieren (Lentsch 2008: 198f.). $\mathrm{Zu}$ den prozessbezogenen Kriterien gehören die Gewährleistung von Transparenz, die Art und Weise der Auswahl der Berater bzw. beratenden Einrichtungen, die Konstituierung und Regelung des Beratungsverhältnisses (Steuerung der Erwartungen), Verlässlichkeit sowie die Ergebnisoffenheit des Verfahrens. Insbesondere die Auswahl und Unabhängigkeit des Beraters und Beratungsprozesses ist ein zentraler Aspekt. Diese wird gewährleistet durch (Weingart/Lentsch 2008: 40, 255; vgl. a. PahlWeber 2011: 398):

- Auswahl des Experten aufgrund von wissenschaftlicher Reputation, 
- Operationale Unabhängigkeit, auch im Hinblick auf die Koordinierung der Forschungstätigkeiten,

- Funktionale Differenzierung zwischen Forschung und Beratung,

- Grad der Formalisierung der Beziehungen zwischen Forschern und Beratenen.

In Bezug auf das Produkt ist einerseits zunächst an jene Kriterien zu denken, die als Gütekriterien wissenschaftlicher Forschung hinlänglich bekannt sind. Neben der Qualität der Daten und der Offenlegung der Wissensbasis betrifft dies auch Fragen der Methodik. Hinzu kommt laut Lentsch (2008) die Qualität in der Formulierung des Beratungsauftrags, der eine klare Aufgaben- und Rollenverteilung enthalten sollte (Abgrenzung, Präzision des Gegenstandes und der Fragestellung). Die Qualität des Produkts erweist sich letztlich aber im Kontext der Anwendung, was andere Prüfverfahren notwendig macht. Eine wie auch immer geartete Qualitätskontrolle müsste das Beratungswissen einem zumindest teilweise öffentlich nachvollziehbaren Bewertungsverfahren unterziehen (Pahl-Weber 2011: 406). Dem steht entgegen, dass Ergebnisse von Beratungsprozessen in der Regel nicht veröffentlicht werden oder in anderer Weise ein peer-review Verfahren durchlaufen. Die Praxis zeigt, dass Beratungswissen eher zwischen Auftraggeber und Auftragnehmer ausgehandelt wird und daher den Charakter eines vertraulichen Berichts behält. Besondere Aufmerksamkeit verlangt daher die Rolle einer wie auch immer gearteten (Fach-)Öffentlichkeit, die in der Lage wäre, Beratungswissen zu bewerten. Aus den USA ist diesbezüglich ein angepasster peer reviewProzess bekannt. Dieses seit längerem diskutierte Verfahren ähnelt dem wissenschaftsinternen peer review-Verfahren, da auch hier sog. peers, also Experten, die nicht zu der zu bewertenden Einrichtung gehören, aber im selben Feld tätig und anerkannt sind, die Leistung bewerten. Aufgrund der genannten Besonderheiten des Beratungswissens müssen die peers aber nicht zwangsweise Wissenschaftler sein, sondern können auch Praxiskontexten oder anderen Beratungseinrichtungen entstammen. Genau hier sieht Lentsch aber die Probleme dieses Verfahrens (2008: 203). Welche Voraussetzungen müssen die peers erfüllen und wie werden sie ausgewählt? Können Beiräte mit Vertretern gesellschaftlicher Interessensgruppen diese Funktion erfüllen und wie müssten sie zusammengesetzt sein? Wie können Interessenskonflikte vermieden werden? Zudem sollte klar sein, dass keine inhaltliche Neubewertung das Ziel des regulatorischen peer reviews sein kann (das wäre letztlich ein Gegengutachten), sondern lediglich die Methodik und die Wissensgrundlage (ebd. 204). In der Regel existiert aber keine fachliche Community, die in einer Weise integriert wäre, die die Herausbildung gemeinsamer professioneller Standards der Politikberatung verspricht, die dann auch als Maßstab herangezogen werden könnten. Wir können festhalten, dass Qualitätssicherung in der wissenschaftlichen Politikberatung in der Raumplanung noch ein Desiderat ist, die Politik selbst aber ein Interesse an einer Qualitätssicherung in der wissenschaftlichen Politikberatung haben sollte. Die Rolle als Auftraggeber und Nutzer macht dies erforderlich. Gleichwohl ist es derzeit nur schwer vorstellbar, über einige allgemeine Kriterien hinaus eine belastbare Systematik zu entwickeln. Dafür ist das Beratungsgeschäft einfach zu vielfältig und die Frage der Qualität der Verwendbarkeit zu vage (Blotevogel/ Wiegand 2015).

\subsection{Grenzarbeit}


Aufgrund der beschriebenen Trends der zunehmenden Verwischung der Grenzen zwischen Wissenschaft und Politik scheint es sinnvoll, das Konzept der „boundary organizations“ bzw. „boundary arrangements“ zu bedenken (Hoppe 2005; Halffman/Hoppe 2005; Straßheim 2013, 2008; Guston 2001). Es handelt sich dabei um hybride Organisationen an der Grenze zwischen Wissenschaft und politischer Praxis, die wichtige vermittelnde Funktionen und Übersetzungsleistungen in einem speziellen Wissensbereich übernehmen, der sich allerdings in der Raumplanung in seiner Struktur von anderen Fachpolitiken wie der Gesundheitspolitik (Krankenkassen und Verbände, die zum Teil wissenschaftliche Institute unterhalten), der Umweltpolitik (SRU, Umweltbundesamt), der Wirtschaftspolitik und der Technologiepolitik unterscheidet (Büro für Technikfolgenabschätzung beim Bundestag) (Pahl-Weber 2011). Grenzorganisationen müssten nicht den Relevanzkriterien beider Systeme (Wissenschaft einerseits, planerische Praxis andererseits) gerecht werden. Mindestens aber müssten sie in der Lage sein, den Transfer existierenden Wissens in die Praxis zu gewährleisten. Umgekehrt könnten sie auf der Grundlage von Konsultationen an der Setzung von Prioritäten für die Forschung mitwirken.

Grenzorganisationen könnten sich auch der Aufgabe der Qualitätssicherung widmen. In Deutschland kommen dafür am ehesten die Akademie für Landesplanung und Raumforschung sowie das Bundesinstitut für Bau-, Stadt- und Raumforschung infrage. Denkbar wäre aber auch, diese Aufgabe in regelmäßigen Abständen in einem Wettbewerbsverfahren neu zu vergeben, um so im Zeitverlauf verschiedene Organisationen zum Zuge kommen zu lassen.

\subsection{Politik- oder Gesellschaftsberatung}

Wünschenswert wäre zudem eine stärkere Differenzierung der öffentlich oder öffentlichkeitswirksamen Wissensproduktion, die der Raumplanung zu mehr Aufmerksamkeit und vielleicht politischer Relevanz verhelfen könnte. Ein enger Begriff von Politikberatung verliert dann seinen Sinn, wenn die Entscheidungen gar nicht von der Politik getroffen werden können, weil sie nicht oder nur begrenzt zuständig ist (Mayntz 1994: 20). Diese Aussage ist für die Raumplanung von besonderer Bedeutung, da viele raumwirksame Entscheidungen von privaten Akteuren getroffen werden. Dies erklärt die Attraktivität einer weiter gefassten Politikder Gesellschaftsberatung für die Raumplanung. Wissenschaftliche Politikberatung in der Raumplanung kann den Trend in Richtung Gesellschaftsberatung durchaus für sich nutzen. Angesichts des bescheidenen politischen Stellenwerts, der der Raumplanung mitunter zukommt, wäre es ein Anliegen, die Raumplanung stärker in gesellschaftspolitische Debatten hineinzutragen. Die in den letzten Jahren zu beobachtenden Entwicklungen in der Schweiz sind, wenn man den Stellenwert der Raumplanung als Thema der Öffentlichkeit bedenkt, sicher bemerkenswert. In der Schweiz hat sich im Vorfeld der Diskussion über das neue Raumordnungsgesetz eine öffentliche Debatte entwickelte, die von Initiativen wie „Darum Raumplanung" und Ausstellungen (road shows) begleitet wurde.

Erforderlich dafür wären Gestaltungsöffentlichkeiten oder organisierte Diskurse zwischen Wissenschaft, Politik und Öffentlichkeit im Sinne von Böschen (2005). Dies wäre Gesellschaftsberatung im Rahmen veranstalteter Öffentlichkeiten, die besonderer Formate 
bedarf. Damit wäre weniger die Politik, sondern die Gesellschaft der Adressat einer als öffentlich verstandenen Wissensproduktion. Eine Distanzierung von der Politik würde dann gar nicht mehr angestrebt. Dies nimmt zugleich den Hinweis von Saretzki auf, der in Anlehnung an USamerikanische Modelle wissenschaftliche Politikberatung als analytisch-deliberativen Prozess sieht (Saretzki 2005). Im Gegensatz zu technokratischen oder dezisionistischen Modellen sieht ein analytisch-deliberativer Prozess eine (sequentielle) Kombination von wissenschaftlichanalytischen Prozessen und Prozessen der Aushandlung der Ergebnisse im Lichte gesellschaftlicher Herausforderungen vor. Die Produktion handlungsrelevanten Wissens verlagert sich mehr in den Planungsprozess (siehe dazu den kritischen Beitrag von BerglundSnodgrass).

\section{5) Zur Rolle des Staates: Wissensproduktion oder Wissensmanagement?}

Abschließend soll eine Einschätzung im Hinblick auf die Notwendigkeit und eventuelle Neuorientierung wissenschaftlicher Politikberatung in der Raumplanung vorgenommen werden. Öffentliche Aufgaben wie die Raumplanung sind in hohem Masse auf externe Expertise angewiesen, die bestimmten Qualitätskriterien entsprechen muss. Erforderlich ist dies, wie oben in Anlehnung an Weingart/Lentsch (2008) dargestellt, aus zweierlei Gründen: zum einen zwingt die sachliche Problemlösung zur Mobilisierung des besten verfügbaren Wissens (epistemische Dimension). Zum anderen erfordert die Notwendigkeit der Legitimation die Rechtfertigung politischer Entscheidungen durch den Rückgriff auf wissenschaftliche Expertise. Angesichts der besonderen Herausforderungen in der Raumplanung und des damit verknüpften Wissensbedarfs stellt sich die Frage nach der staatlichen Verantwortung für die Wissensproduktion und die Gestaltung der Grenzarbeit (Voßkuhle 2008: 21). Wie kann der offenbar wachsende Bedarf an Expertise angesichts der genannten Trends gewährleistet werden? Sollen staatliche Einrichtungen oder öffentlich finanzierte Forschungseinrichtungen nahe an der Exekutive selbst forschen oder nur den Wissensbestand managen und auf die Qualitätssicherung achten? Wie dargestellt hat sich das System der Produktion politisch relevanten Wissens nicht nur im Bereich der Raumplanung im Hinblick auf die Akteure und Formate ausdifferenziert. Pahl-Weber weist darauf hin, dass der Anteil an externer Forschung, der durch das Bundesinstitut für Bau-, Stadt- und Raumforschung vergeben und moderiert wird, im Vergleich zu anderen Ressortforschungseinrichtungen recht hoch ist (Pahl-Weber 2011: 404). Angesichts eines Überangebots an handlungsrelevantem Wissen, das in zum Teil hoher Qualität mit unterschiedlichen Intentionen in die öffentliche Diskussion eingebracht wird, scheint eine Art Meta-Kompetenz der Wissensselektion notwendig zu sein, was klare Relevanzund Gütekriterien erfordert. Hier sind auch Organisationsfragen berührt (siehe Beitrag von Feiertag). Eine mögliche Reaktion wäre der gezielte Einsatz von ggf. gestuften Wettbewerbsverfahren durch staatliche Wissensagenturen, an denen neben privaten und öffentlichen Forschungseinrichtungen vielleicht auch zivilgesellschaftliche Organisationen teilnehmen könnten. Zugleich macht die Vielfalt der Wissensquellen dauerhafte Formen der Politikberatung durch Beiräte und Netzwerkorganisationen wie die Akademie für Raumforschung und Landesplanung unverzichtbar. 


\section{Literatur}

Altrock, Uwe 2016: Evaluation komplexer Programme in der Städtebauförderung, in: Informationen zur Raumentwicklung, Heft 1 /2016, 5-16.

Aring, J./ Sinz, M. 2006: Neue Leitbilder der Raumentwicklung in Deutschland. In: DISP 42: 165, 43-60.

BBAW / Berlin-Brandenburgische Akademie der Wissenschaften 2008: Leitlinien Politikberatung. Berlin

Beck, U. / Bonß, W. 1989: Verwissenschaftlichung ohne Aufklärung? In: dies. (Hrsg.), Weder Sozialtechnologie noch Aufklärung. Analysen zur Verwendung sozialwissenschaftlichen Wissens. Frankfurt: Suhrkamp, 7 - 46.

Boghrat, J./ Weber, M./ Zimmermann, K./Lamping, W. 2014: Kommunalverwaltung und Klimawandel Wissensgenerierung, Framing, und die Erzeugung von Handlungsfähigkeit, in: Der moderne Staat, 7. Jg., Heft 2, 289-309.

Blotevogel, Hans-Heinrich 2005: Raumordnung in den 1960er und frühen 1970er Jahren: die Blütezeit der räumlichen Gesamtplanung: in: Akademie für Landesplanung und Raumforschung (Hrsg.) Grundriss der Raumplanung, Hannover 134-167.

Blotevogel, Hans-Heinrich/Wiegand, Timm Sebatian 2015: Zur Evaluation von Wissensgenerierung und Wissenstransfer in der Akademie für Raumforschung und Landesplanung (ARL) - Leibniz-Forum für Raumwissenschaften, in: Raumforschung und Raumordnung, Vol. 73: 155-165.

Böcher, M. 2007: Wissenschaftliche Politikberatung und politischer Prozess, in: Max Krott/Michael Suda (Eds.), Macht Wissenschaft Politik? Erfahrungen aus der wissenschaftlichen Beratung im Politikfeld Wald und Umwelt. Wiesbaden: VS Verlag für Sozialwissenschaften. 14-42.

Böschen, S. 2005: Reflexive Wissenspolitik. Formierung und Strukturierung von Gestaltungsöffentlichkeiten, in: Bogner, A./ Torgersen, H. (Hrsg.): Wozu Experten? Ambivalenzen der Beziehung von Wissenschaft und Politik, Wiesbaden: VS Verlag für Sozialwissenschaften, 241-266.

Bröchler, Stephan/ Schützeichel, Rainer (Hrsg.) 2008: Politikberatung. UTB: Lucius \& Lucius.

Davoudi, S. 2006: The evidence - policy interface in strategic waste planning for urban environments: the technical and the social dimensions. In: Environment and Planning C: Government and Policy, Vol. 24, 681-700.

de Roo, Gert/ Jean Hillier/ Joris van Wezemael 2012: Complexity and Planning: Systems, Assemblages and Simulations, Farnham, U.K. and Burlington, Vermont: Ashgate Publishing.

Döhler, M./Bach, S. 2012: Mandated science and the problem of neutral expertise - The case of governmental research agencies, WZB Discussion Paper SP III 2012-602, Berlin.

Falk, S./ Rehfeld, D./ Römmele A./ Thunert, M. 2006 (Hrsg.): Handbuch Politikberatung. Wiesbaden.

Faludi, A./ Waterhout, B. 2006: Introducing evidenced-based planning. In: disp, vol. 42, issue 165, 4-13.

Fischer, F. 2009: Democracy and Expertise. Reorienting Policy Inquiry. Oxford.

Fürst, D. 2003: Paradigmatische Steuerung in der Regionalplanung. In: Fürst, D./ Löb, S./Rudolph, A./ Zimmermann, K.: Steuerung durch Regionalplanung Baden-Baden: Nomos, 125-142.

Fürst, D. 2010: Raumplanung. Herausforderungen des deutschen Institutionensystems. Detmold.

Geiss, Anna 2008: Mediationsverfahren als alternative Form der Politikberatung, in: Bröchler, Stephan/ Schützeichel, Rainer (Hrsg.) 2008: Politikberatung. UTB: Lucius \& Lucius. 130-146.

Gieryn, T. F. 1983: Boundary-Work and the Demarcation of Science from Non-Science. Strains and Interests in Professional Ideologies of Scientists. In: American Sociological Review 48 (6), 781-795.

Gieryn, T. F. 1998: Cultural Boundaries of Science. Credibility on the Line, Chicago/London.

Göddecke-Stellmann, J. 2011: Politikberatung in der Raum- und Stadtentwicklung, In: Informationen zur Raumentwicklung H 7/8.2011, I-V.

Gramelsberger, G. 2009. Simulation - Analyse der organisationellen Etablierungsbestrebungen der (neuen) epistemischen Kultur des Simulierens am Beispiel der Klimamodellierung, Jost Halfmann, Falk Schuetzenmeister (Hrsg.): Organisationen der Forschung. Der Fall der Atmosphärenwissenschaften, Wiesbaden: VS Verlag für Sozialwissenschaften, 30-52.

Guston, David H. 2001: Boundary Organizations in Environmental Policy and Science: An Introduction, in: Science, Technology, \& Human Values, Vol. 26, No. 4, Special Issue: Boundary Organizations in Environmental Policy and Science, 399-408.

Hoppe, R. 2005: Rethinking the science-policy nexus: from knowledge utilization and science technology studies to types of boundary arrangements. In: Poiesis, 3, 199-215. 
Jasanoff, S. 2005: Designs on Nature: Science and Democracy in Europe and the United States. Princeton (NJ): Princeton University Press.

Katz, Bruce/Bradley, Jennifer 2013: The Metropolitan Revolution: How Cities and Metros Are Fixing our Broken Politics and Fragile Economy (Brookings Focus Book) Washington.

Knapp, Gert-Jan/Nedovic-Budic, Zorica/Carbonell, Armando 2015: Planning for states and nation-states in the U.S and Europe. Lincoln Institute of Land Policy: Cambridge.

Korinek, R.-L.; Veit, S. 2013: Wissenschaftliche Politikberatung als Grenzarbeit. Ein Konzept zur Analyse institutionalisierter Beratungsformen in Politikfeldern. In: Der moderne Staat (Sonderheft 1), 261-284.

Lendi, M. 2005: Politikberatung. Nachfrage, Resonanz, Alibi. Zürich: vdf, Hochsch.-Verl. an der ETH.

Maasen, S./ Weingart, P., 2005: What's New in Scientific Advice to Politics. In: dies. (Hrsg.),

Democratization of Expertise? Exploring Novel Forms of Scientific Advice in Political Decision-Making, Berlin/Heidelberg/New York, 1-19.

Mayntz, R. 2006: Die Organisation wissenschaftlicher Politikberatung in Deutschland. In: Heidelberger Akademie der Wissenschaften (Hrsg.): Politikberatung in Deutschland. 1. Aufl. Wiesbaden, 115-122.

Mayntz, R. 1994: Politikberatung und politische Entscheidungsstrukturen. Zu den Voraussetzungen des Politik-beratungsmodells. In: Axel Murswieck (Hrsg.): Regieren und Politikberatung. Opladen: Leske + Budrich, 17-29.

Nowotny, H./ Scott, P./ Gibbons, M (Hrsg.) 2001: Re-thinking science: knowledge and the public in an age of uncertainty. London: Polity-Press.

Nuissl, H. 2000: Erschließungsaufgaben. Die Verwendung sozialwissenschaftlichen Wissens in der räumlichen Planung. Berlin.

Pahl-Weber, Elke (2011): Politikberatung in der Ressortforschung zur Bau-, Stadt- und Raumforschung. In: Informationen zur Raumentwicklung (7/8), 397-408.

Rydin, Y. 2007: Re-examining the role of knowledge within planning theory. In: Planning Theory 6, 1, 5268.

Saretzki, T. 2005: Welches Wissen - wessen Entscheidung? Kontroverse Expertise im Spannungsfeld von Wissenschaft, Öffentlichkeit und Politik. In: Bogner, A./ Torgersen, H. (Hrsg.): Wozu Experten? Ambivalenzen der Beziehung von Wissenschaft und Politik, Wiesbaden: VS Verlag für Sozialwissenschaften, 345-369.

Saretzki, T. 1997: Demokratisierung von Expertise? Zur politischen Dynamik der Wissensgesellschaft. In: Klein, A.; Schmalz-Bruns, R. (Hrsg.) 1997: Politische Beteiligung und Bürgerengagement in Deutschland. Möglichkeiten und Grenzen, Bonn: Bundeszentrale für politische Bildung und Baden-Baden: Nomos, 277-313.

Schelsky, H. 1965: Der Mensch in der wissenschaftlichen Zivilisation, in: ders. Auf der Suche nach Wirklichkeit. Düsseldorf: Diederichs, 439-480.

Schneidewind, U./Singer-Brodowsky, M. 2013: Transformative Wissenschaft. Klimawandel im deutschen Wissenschafts- und Hochschulsystem Marburg, Metropolis-Verl.

Schützeichel, Rainer 2008: Beratung, Politikberatung, wissenschaftliche Politikberatung, in: Bröchler/Schützeichel, 5-32.

Sinz, M. 2011: Raumordnung als Gegenstand von Politikberatung - ein Erfahrungsbericht. In: Informationen zur Raumentwicklung, H 7/8, Bonn, 471-486.

Straßheim, H. 2008: Kulturen der Expertise und politischen Wissensproduktion im Wandel: vergleichende Beobachtungen, in: Gosewinkel, D./ Schuppert, G. F. (Hrsg.): Politische Kultur im Wandel von Staatlichkeit. Berlin: Edition Sigma, 303-316.

Straßheim, H. 2013: Politische Expertise im Wandel: Zur diskursiven und institutionellen Einbettung epistemischer Autorität, in: Kropp, S./Kuhlmann, S. (Hrsg.): Wissen und Expertise in Politik und Verwaltung, Der moderne Staat, Sonderheft 1/2013, 65-87.

Voßkuhle, Andreas 2008: Das Konzept des rationalen Staates, in: Schuppert, Gunnar Folke/ Voßkuhle, Andreas (Hrsg.) Governance von und durch Wissen, Nomos: Baden-Baden. 13-32.

Weingart, P./ Lentsch, J. 2008: Wissen, Beraten, Entscheiden. Form und Funktion wissenschaftlicher Politikberatung in Deutschland. Weilerswist: Velbrück.

Zimmermann, K. 2010: Der veränderte Stellenwert von Wissen in der Planung, in: Raumforschung und Raumordnung, Heft 2, 115-125. 
